\title{
Movement and the judged duration of visual targets
}

\author{
WILLIAM T. LHAMON and SANFORD GOLDSTONE \\ Cornell University Medical College, New York, New York 10021
}

\begin{abstract}
Two experiments studied the effects of movement of visual patterns and visual stimulus change without movement upon judged duration. A horizontally moving grating pattern was judged longer than the same stationary pattern. Similarly, a stationary flickering light was judged longer than a stationary steady light. In visual processing of temporal magnitude information, stimulus change appears to represent a basic factor. Stimulus change produces longer judged duration.
\end{abstract}

Prior studies (e.g., Goldstone \& Goldfarb, 1964; Goldstone \& Lhamon, 1972) demonstrated that short sounds were judged longer than lights upon direct comparison. Further investigation (Goldstone \& Lhamon, 1974) showed that static light patches were judged shorter than others in motion; moving line patterns consisting of a triangle waves, Lissajous figures, and random wave forms were judged longer than solid lights while the same Lissajous figure without motion was no different than the solid patch.

Two experiments were conducted to learn more about movement and judged visual duration. The first compared directly a stationary pattern (i.e., Schade grating) with the same pattern in motion; earlier studies (Goldstone \& Lhamon, 1974) compared stationary or moving patterns with solid lights separately with the movement effect inferred. This experiment was a direct attempt to confirm the movement effect. The second experiment compared flickering with steady lights to determine if the movement effect required positional changes of line patterns or merely stimulus change to increase judged visual duration.

\section{EXPERIMENT I}

This experiment tested directly the hypothesis that a moving line pattern (Schade grating) would be judged longer than the same stationary pattern. Using pair comparison, subjects judged the duration of the last member of each pair as longer or shorter than the first; the pairs consisted of either two moving durations, two stationary durations, a moving followed by a stationary duration, or a stationary followed by a moving duration.

\section{Method}

Apparatus. Durations were recorded on magnetic tape which controlled timing of (1) moving and stationary visual stimuli, (2) interstimulus interval, and (3) interpair interval. The tape contained 105 pairs of durations. The first of each pair, or standard, was a 1.00 -sec duration followed by a variable of .70, $.85,1.00$, or $1.30 \mathrm{sec}$; interstimulus and interpair intervals were 2.00 and $10.00 \mathrm{sec}$, respectively. The system produced durations

Requests for reprints should be sent to William T. Lhamon, Edward W. Bourne Behavioral Research Laboratories, New York Hospital-Cornell Medical Center, 21 Bloomingdale Rd., White Plains, New York 10605. with a constant error of +10 to $+24 \mathrm{msec}$ with repeat accuracy of $.15 \%$. Five pairs were practice and 100 were distributed to permit five presentations of each stimulus sequence (moving-moving, stationary-stationary, moving-stationary, stationary-moving) and five standard-variable combinations.

The pattern was either a stationary Schade (1956) grating or the same grating in left-to-right horizontal motion at 10 lines/sec on an $8 \times 10 \mathrm{~cm}$ oscilloscope tube face with viewing distance of $208 \mathrm{~cm}$; the grating consisted of vertical light and dark bars $.8 \mathrm{~cm}$ wide given a spatial frequency at viewing distance of 2.4 cycles per degree with 6.25 cycles visible on tube face. Luminance of the pattern was $2.19 \mathrm{~cd} / \mathrm{m}^{2}$ with ambience at $.20 \mathrm{~cd} / \mathrm{m}^{2}$; contrast of the grating was adjusted to maximum (about 90\%).

Subjects. Ten adult volunteers age 20 to 48 years.

Procedure. Subjects judged the second variable member of each pair as shorter or longer than the first 1.00 -sec standard using a 5-category scale: 1 , shorter; 2 , slightly shorter; 3 , equal; 4, slightly longer; 5, longer.

An Average Category Response (ACR), or mean response for every condition was computed. Analysis of variance included moving-or-stationary-judged, same-or-different-stimuli-paired, and variable-durations as factors; the same-or-different factor pertained to whether subject judged moving-moving or stationary-stationary (same), or, stationary-moving or moving-stationary (different).

\section{Results}

The left panel of Figure 1 displays the mean ACR by Durations plots for the significant component of the Same-Different by Moving-Stationary-Judged interaction $(\mathrm{F}(1,9)=18.46, \mathrm{p}<.001)$, which shows that moving durations were judged longer than static durations upon direct comparison; nine of ten subjects showed this effect. The moving-moving and stationary-stationary judgments did not differ signficantly from each other and, if shown, would be sandwiched between the displayed curves. The movement effect was obtained directly, confirming the speculation by Goldstone and Lhamon (1974).

\section{EXPERIMENT II}

This experiment tested the hypothesis that stimulus change, not apparent travel across space, is the property of movement that increases judged visual duration. Subjects compared stationary steady lights with stationary flickering lights. 


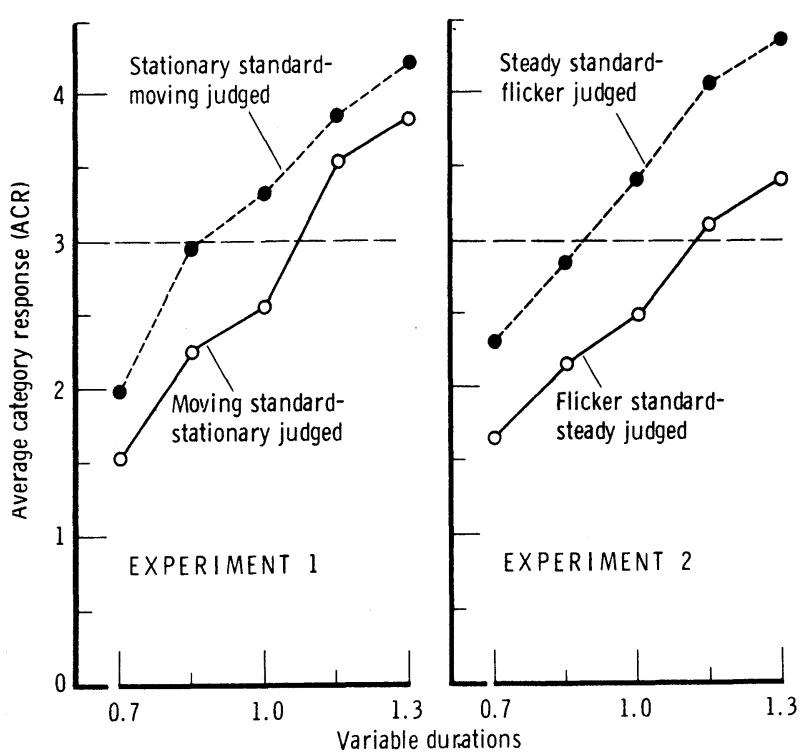

Figure 1. Left panel, Experiment I: Mean average category response (ACR) by Variable Durations plots for the stationary standard-moving judged and moving standard-stationary judged conditions; $\mathbf{N}=10$ for each point. Right panel, Experiment II: Mean ACR by Durations plots for the steady standard-flicker judged and flicker standard-steady judged conditions; $N=5$ for each point.

\section{Method}

Apparatus. The source was a light emitting diode presented either at $100 \mathrm{~Hz}$ (steady) or $10 \mathrm{~Hz}$ (flicker); the diode appeared as a circular red target of $5.08 \mathrm{~mm}$ diam at viewing distance of $76.2 \mathrm{~cm}$.

Subjects. Five adult volunteers age 21 to 37 years.

Procedure. The procedure was identical with that of the first experiment with subjects judging pairs of lights that were flicker-flicker, steady-steady, flicker-steady, or steady-flicker five times for each standard-variable combination. Analysis of variance for ACR measures included flicker-or-steady judged, same-or-different-stimuli-paired, and variable-durations as the factors.

\section{Results}

The right panel of Figure 1 displays the mean
ACR-by-durations plots for the significant component of the Same-Different by Ficker-Steady-Judged interaction $(\mathrm{F}(1,4)=33.92, \mathrm{p}<.005)$ which shows that flickering lights were judged longer than steady upon direct comparison; all five subjects showed this effect. The flicker-flicker and steady-steady judgments did not differ significantly from each other and were between the displayed curves. These results are similar to those of the first experiment and support the hypothesis that stimulus change was the basic factor in increased judged visual duration accompanying linear movement.

\section{DISCUSSION}

The auditory and visual systems differ in their reckoning of temporal information (Goldstone \& Lhamon, 1972). In processing the amount of time, intensity seems to be a more important factor for the auditory system than for the visual, while movement was implicated with vision (Goldstone \& Lhamon, 1974). These studies confirm the prior implication of visual movement upon temporal processing with direct comparison of moving and stationary gratings. The fact that an identical effect was produced by a stationary flicker suggests that stimulus change alone, without positional variation, represent the property of movement that increases judged visual duration. Now we are left with questions about the nature and shape of this stimulus change-judged duration function. Further exploration employing pair comparison and flicker varying frequency and light-dark ratio should be useful, and moving patterns can be investigated more fully with varied rate, spatial frequency, and spatial orientation.

\section{REFERENCES}

Goldstone, S., \& Goldfarb, J. L. Direct comparison of auditory and visual duratons. Journal of Experimental Psychology, $1964,67,483-485$.

Goldstone, S., \& Lhamon, W. T. Auditory-visual differences in human temporal judgment. Perceptual and Motor Skills, 1972, 34, 623-633.

Goldstone, S., \& Lhamon, W. T. Studies of auditory-visual differences in human time judgment: 1 . Sounds are judged longer than lights. Perceptual and Motor Skills, 1974, 39, 63-82.

Schade, O. H. Optical and photoelectric analog of the eye. Journal Optical Society of America, 1956, 46, 721-739.

(Received for publication October 16, 1974.) 